\title{
Construction of Knowledge Questionnaire Regarding Junk Food Consumption
}

\author{
Suchandra Samanta Mondal ${ }^{1}$, Santosh Mukherjee ${ }^{2}$, Deb Prasad Sikdar ${ }^{3}$ \\ ${ }^{1}$ Assistant Professor, Krishnanagar B.Ed. College, Nadia, West Bengal, India \\ ${ }^{2}$ Vice-Principal, Krishnanagar B.Ed. College, Nadia, West Bengal, India \\ ${ }^{3}$ Professor, Department of Education, University of Kalyani, Nadia, West Bengal, India
}

Corresponding Author: Deb Prasad Sikdar

\begin{abstract}
Aim: The present paper aims to discuss on the construction and validation of a survey tool for measuring knowledge of people of West Bengal regarding junk foods.

Method: Questionnaire was selected as the data collection tool. The questionnaire was prepared on the basis of certain dimensions and initially it had 41 items. At first, the questionnaire was administered to a target population of 30 respondents. After the pre-test among 30 respondents the readability, discrimination indices, and difficulty indices were determined for 'Knowledge' questionnaire. Rejection criteria were set for each parameter and the final questionnaire was constructed with the items retained after validation. The reliability of the tool was confirmed by TestRetest method.

Result: Finally, the knowledge questionnaire retained 31 items. Reliability co-efficient was found to be 0.76090 which was significant.

Conclusion: Validity and reliability of the knowledge questionnaire were well established. There were 31 items in the final questionnaire depending on the rejection criterion. The high reliability of the questionnaire was found. This questionnaire will help to find out the level of knowledge of people regarding junk food and its effects on health.
\end{abstract}

Key Words: Junk food, Food consumption, Knowledge

\section{INTRODUCTION}

Worldwide food selection and consumption pattern have increased a lot towards readymade foods within a couple of decades. Exercise of brain has increased but normal physical exercise has got diminished. The consequences of these two have increased various health risks in our children, young adults and adults, in the form of reduction in stamina, obesity, juvenile diabetes, high blood pressure, etc. Their educational level also may have effects in this regard. ${ }^{[1]}$ Eating frequent junk foods causes teens and young adults to gain more weight and they face an increased risk of developing obesity. Childhood obesity has more than tripled in the last three decades. The occurrence of obesity among adolescents has increased from 5 to $18 \%$. ${ }^{[2]}$ As per National Institute of Nutrition (NIN) "junk foods" are defined as "those containing little or no proteins, vitamins or minerals but are rich in salt, sugar, fats and are high in energy (calories)". Artificially aerated drinks, ice creams, potato chips, chocolate, French cries are some examples of Junk food. ${ }^{[3]}$ A study by Sharma ${ }^{[4]}$ suggested that junk food consumption alters brain activity in a manner similar to addictive drugs like cocaine or heroin. After many weeks with unlimited access to junk food, the pleasure centers become decentralized, requiring more food for pleasure. The reasons behind 
the junk food habit may be not enough time for food preparation at home, may be the easy availability or addictive nature of the junk food. ${ }^{[5]}$ Sedentary life style of the people also may be behind such habit. ${ }^{[6]}$

The National Restaurant Association of India, 2010 has reported that in India the fast food industry is growing at a compound annual growth rate of $35-40$ percent. Rs.6750-Rs. 8000 crore is the estimation of this industry. In India, according to Food Safety Standard Authority of India (FSSAI) junk food is not defined, rather it is included within the proprietary food category- not standardized under regulations. This category of food is only expected to declare their composition or nature of food and comply with general regulations under the food act. ${ }^{[7]}$ A survey in Delhi revealed that most adolescent girls highly prefer the fried food and sweet snacks and parents and teachers were found most influential determinants for such food choice. ${ }^{[8]}$ In some rural areas also $80 \%$ of the people including adolescents have the knowledge regarding the calories of junk food but do not know the nutritional contents properly. [9] In West Bengal, obesity among the children has been reported alarmingly increased. Some of the reasons behind such problem may be due to lack of physical activities, more restaurant and fat intake as mentioned by some pediatricians. ${ }^{[10]}$ Recently University Grant Commission has given a direction to all Universities and Higher Educational Institutions for banning the junk food sale on their campuses. ${ }^{[11]}$ In West Bengal some Universities are also taking actions for banning Junk food on their campuses. ${ }^{[12]}$

Dietary behaviour is important for the development and growth of human beings. Ideally a person should have healthy food habit with minimum or no consumption of junk food. The person should have proper knowledge regarding nutrition and junk food to avoid junk food consumption. Therefore, it is necessary to find out the real scenario of knowledge of people of West Bengal regarding junk food consumption. The study may help people to select better food and consumption behavior, may aware and sensitize them against the junk foods.

Designing and construction of research tools is the essential step for any research study to be conducted systematically. When a questionnaire has same questions and same coding system, it can be said as standardized questionnaire. ${ }^{[13]}$ In the present study the details regarding the construction of the tools needed for the survey research and their validation has been described. The main objective of this paper is to construct knowledge questionnaire for measuring the knowledge of the people of West Bengal regarding junk food consumption.

\section{MATERIALS AND METHODS}

Though there are many types of survey tools at present, researchers generally use the interviews and questionnaires for data collection. ${ }^{[14]}$ In the present study, questionnaire was selected and designed for collection of data. The target population was adolescents (15-21), young adults (22-35) and adults of the West Bengal. Knowledge is the understanding of any given topic. ${ }^{[15]}$ The options of each item were arranged in such a way that if the score increases it will contribute to the increase in the overall score of knowledge (K)-score. The $\mathrm{K}$-score of a subject is expected to increase with the knowledge of junk food and his/her distinguishing power between nutritious food and junk food. It is expected that if a subject has greater $\mathrm{K}$-score then he/she will show less tendency of consuming junk food. The dimensions like identifying the junk food, knowledge of nutrition, junk food and health problems due to junk food were taken for the knowledge scale.

\section{Dimensions}

The questionnaires were formulated by keeping in mind that the participants would have to ponder over each option and in no case the answer was directly 
embedded in the question itself. The item numbered K.1 contained 16 foods and the question was to categorize them under two food categories (i) Healthy food and (ii) Junk food. Thus there were 16 question units. Multiple options were correct. "Yes/No" - type of options were avoided. Open ended question could have fetched more accurate result of knowledge of a subject regarding junk food, but it would have been more difficult to quantitate.

\section{a) Knowledge of Nutrition: \\ i) The physiological function of food groups}

In this section four items K.2, K.3, K.4 and K.5 were enlisted to know whether the respondents know the physiological functions of different food groups. In question K.2 two options were correct. If a subject was able to identify both the options the points secured would be 02. Multiple correct options made the choice making slightly difficult for a subject. It inserts a certain degree of uncertainty. Thus it was expected to produce greater degree of discrimination from this type of questions. The rest of the questions ranging from K.3 to K.5, all had single correct option.

ii) The comparison of foods in terms of specific nutrient content

Each subject should know that which food item contains which nutrients like fat, carbohydrate, protein, vitamins and minerals. K.6 and K.7 contain a single correct option each. K.8 item comprises of three options (i) Yes, (ii) No and (iii) I don't know. The correct option was 'Yes'. When a subject selected the option 'Yes' the score taken was one, otherwise both the incorrect options fetched zero.

\section{iii) Balanced diet}

Two questions K.9 and K.10 were created to check the knowledge of balanced diet. In K.9 three options of servings were given.

\section{b) Junk food and health problems}

A subject should know the problems associated with junk food consumption.
This was included in the domain of knowledge. Seven items ranging from K.11 to K.17 were given, out of which K.11 itself contained 7 correct question units and hence the highest possible score of seven. K.13 had three correct options with a maximum possible score of three and rest of the items had single correct option each.

\section{Item pool for knowledge Questionnaire}

At the beginning of the development and standardization of the Knowledge questionnaire the advice, guidance and instruction of the respectable guide were followed. The view of the experts working in different fields like health education, medicine, education were taken for authentication of the different dimensions of the questionnaire. According to their valuable suggestions the test items were corrected, rejected and added. Ultimately the knowledge questionnaire retained 31 question units.

\section{Pre-Try Out of the Knowledge Questionnaire}

A total of 30 respondents were chosen among friends, family and acquaintances to get the intended range in age (15-21yrs., 2235 yrs., 35yrs. and above), gender (male and female), socio economic status, educational background (Primary, secondary, higher education) and covering different rural and urban areas of West Bengal in the test population. The items were prepared in two languages, Bengali and English. The questionnaires were given to the respondents in written form. Respondents were asked to fill out the questionnaire in relaxed pace. They were asked to note the start time and the end time to obtain the time required for answering the questionnaire. After completion the respondents were asked to note their experience of answering the questionnaire with the help of five options (i) Very easy (ii) Easy (iii) Normal (iv) Difficult (v) Very difficult. From the pie-chart (Fig. 1) it is evident that majority of the respondents found the questionnaire not difficult at all. 


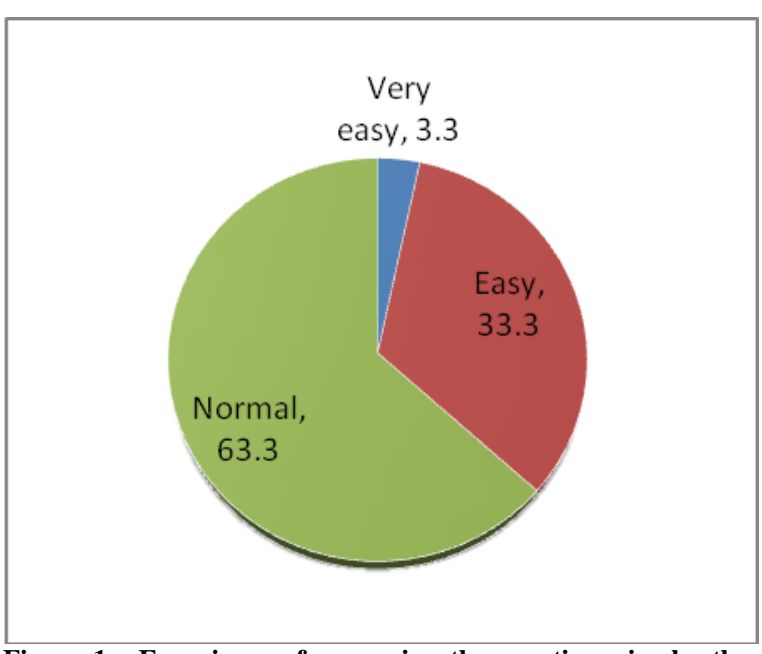

Figure 1: Experience of answering the questionnaire by the respondent

After the collection of data, they were analyzed for edition of the individual question and statement. The experts also suggested for further language improvement and getting rid of any complexity, repetition or ambiguity in the test items.

\section{Try Out of the Knowledge Questionnaire}

The questionnaire was then administered on 30 respondents of rural and urban areas of West Bengal. The questionnaires were distributed by the researcher to the respondents. The respondents were requested to put tick $(\sqrt{ })$ mark against each test item according to the instructions given. The score of individual respondent was made against each individual test item.

\section{Validity and reliability of the Knowledge Questionnaire}

The validation of the knowledge questionnaire included the four major considerations. At first, the content was validated. Authentication of the different dimensions was assured by the experts. They gave their valuable suggestions on inaccuracy, repetition, ambiguity, irrelevance, of the test items, according to which the items were rejected, altered and necessary changes were done. The content of knowledge questionnaire was found covering the essential knowledge regarding junk food and the test items were according to the respective purposes of the research.
Thus the items were made appropriate for the adolescents, middle age and above 35 years age group of people of West Bengal. The Construct validity of the questionnaire was determined by the experts about whether the test items could measure the type of knowledge regarding junk food intended to be measured. The difficulty, ease and the other factors that may positively or negatively influence the respondents during completion of the test were also determined. The test items in the knowledge questionnaire were found relevant in the present situation of junk food consumption and its health consequences. Thus the face validity of the knowledge questionnaire was established.

\section{STATISTICAL ANALYSIS \\ Flesch-Kincaid readability test}

The Flesch-Kincaid readability test was employed to measure the difficulty level of a sentence in English to understand it. MS Word® 2007 (Microsoft Inc.) was used for calculating Flesch Reading Ease and Flesch-Kincaid Grade Level. ${ }^{[16]}$ The language of the questions was found understandable to persons with knowledge of $8^{\text {th }}$ standard. The average of the total 16 test items was found to be 7.4 and the maximum and minimum "Flesch-Kincaid Grade Levels" were found to be 11.6 and 2.2 respectively. The range of "FleschKincaid Grade Level" may be expressed as $7.4 \pm 2.8$ (SD). Therefore, the language was easy to read and it may be taken as Plain English, easily understood by 13- to 15year-old students.

\section{Item analysis of the Knowledge Questionnaire}

Item analysis helps to select or omit an item in a test ultimately improving the item. ${ }^{[17]}$ Here the difficulty index and discrimination index were carried out for the item analysis.

a) Determination of difficulty index of each item of Knowledge questionnaire

The difficulty index of an item is defined as the proportion of respondents 
giving correct answer to that particular item. The difficulty index of the knowledge questionnaire was calculated and the items having difficulty indices within 20 to $80 \%$ were retained in the final questionnaire, rest of the items were rejected.

\section{b) Calculation of discrimination index of Knowledge questionnaire}

Discrimination index is a parameter that shows the sensitivity of the knowledge tool. The respondents were grouped into upper and lower-scoring groups of equal size. First the total score of each candidate was calculated by summing the scores of each item and then the rows were sorted by the total score. The respondents were divided into two groups of upper $27 \%$ of the candidates (i.e. 8 nos,) and the lower $27 \%$ (i.e. 8 nos.). The proportion of the respondents responding correctly pertaining to a particular item in the lower scoring group $\left(\mathrm{P}_{\mathrm{L}}\right)$ is subtracted from the proportion of the candidates responding correctly in the upper-scoring group $\left(\mathrm{P}_{\mathrm{U}}\right)$.

The guideline for interpretation of Dvalues prescribed by Ebel ${ }^{[18]}$ was adopted to reject or accept an item. Items with large
D-values signified large differences in the proportion of correct responses between two groups. Larger D values are suitable for inclusion in the final tool while items with smaller or negative D-values were not.

\section{Reliability of the Knowledge Questionnaire}

In the present study the test-retest method has been followed to determine the reliability of knowledge scale. The total score of a respondent was summed up and the score was normalized by calculating the percentage with respect to the highest possible score, i.e. 41 for the knowledge tool. Same questionnaire was given to a respondent twice but after an interval to assess whether a respondent gives the same answer again. The time gap between the test (T1) and retest (T2) was 30 days. Correlation coefficient was calculated from the paired scores of the respondents (i.e. T1 and $\mathrm{T} 2$ ). Correlation between $\mathrm{T} 1$ and $\mathrm{T} 2$ scores was found to be $\mathrm{R}=0.76090$. Fig. 2 shows the correlation between the two scores.

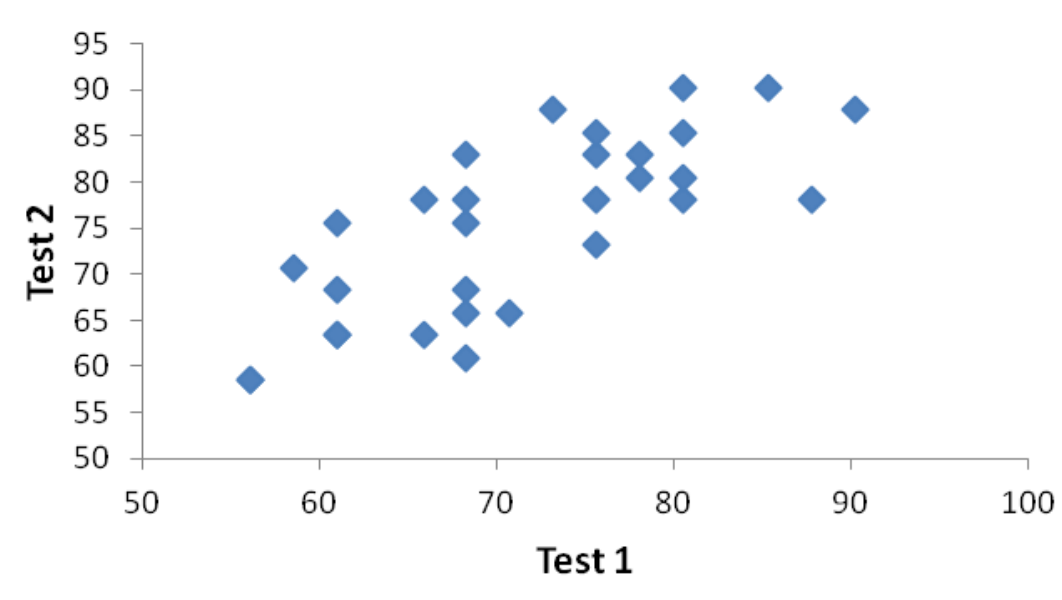

Figure 2: Reliability of $\mathrm{K}$-score $(\mathrm{R}=0.76090)$

\section{RESULT}

Finally, among 17 test items in the Knowledge questionnaire, 16 test items against 3 dimensions like identifying junk food, Knowledge of Nutrition, Junk food and health problems were retained. The item numbered K.1 contained 7 junk foods i.e., correct options and so there were 7 question units or sub-items. The item numbered K.2 and K.10 and K.12 had 2, 7 and 3 correct 
options and therefore, 2, 7 and 3 question units or sub-units. The rests test items have single correct option. Thus there were total 31 question units in the knowledge questionnaire. Distribution of the test items among different dimensions of knowledge questionnaire regarding junk food has been shown in the table 1 .

\begin{tabular}{|l|l|l|l|}
\hline \multicolumn{3}{|c|}{ Table 1: Distribution of the test items among different dimensions of Knowledge questionnaire regarding Junk food } \\
\hline $\begin{array}{l}\text { Serial } \\
\text { no. }\end{array}$ & Dimensions & Items/Statement no. \\
\hline 1 & Identifying the junk food & K1.(07 question units) & question units \\
\hline 2 & Knowledge of nutrition & $\begin{array}{l}\text { The physiological function of } \\
\text { food groups }\end{array}$ & K2 (02 question units), K3, K4, K5 \\
\cline { 3 - 4 } & $\begin{array}{l}\text { The comparison of foods in } \\
\text { terms of specific nutrient } \\
\text { content }\end{array}$ & K6, K7, K8 \\
\cline { 3 - 5 } & Balanced diet & K9 & $\begin{array}{l}\text { K10 (07 questions), K11, K12 (03 question units), } \\
\text { K13, K14, K15, K16 }\end{array}$ \\
\hline 3 & Junk food and health problem & Total \\
\hline
\end{tabular}

\section{DISCUSSION}

The aim of the present study was the construction of knowledge questionnaire regarding junk food. It was done systematically and logically. Validity and reliability of the questionnaire were found well established. More or less similar type of study was found where a structured knowledge questionnaire regarding harmful effects of Junk food was constructed consisting of 30 test items. Experts from the field of community health nursing, child health nursing and nutrition confirmed the content validity of the questionnaire. Sample for pilot study were 10 adolescent students. Reliability of the questionnaire was determined by Karl Pearson's Correlation coefficient and it was $0.78 .{ }^{[4]}$ In another study, knowledge questionnaire along with questionnaire on attitudes and practices on nutrition were constructed and an expert panel scrutinized the content validity. Here, number of questions was reduced by Exploratory Factor Analysis (EFA). Dimensions were somewhat similar with the present study, like, food recommendation, nutrients related to diseases and healthy diet. Questionnaire contained multiple-choice questions. ${ }^{[19]} \mathrm{A}$ nutrient knowledge questionnaire for adults was developed by Parmenter and Wardle in 1999. In this study, the construct validity of the questionnaire was established and for reliability the test-retest method was employed. Reliability was higher than the present study. ${ }^{[20]}$ Another research work shows eight steps for knowledge questionnaire development. Here, construct validity, face validity and content validity were determined to establish the validity of the questionnaire as was done in the present study. ${ }^{[21]}$ The questionnaire constructed in the present study may be used in other related future research works.

\section{CONCLUSION}

The purpose of the questionnaire construction was to collect data on the knowledge of the people regarding junk food. Adolescents (15-21), young adults (22-35), and adults of the rural and urban areas of West Bengal were the target population. At first, there were 41 items in the questionnaire. There were single and multiple correct options. The main dimensions on the basis of which the test items constructed were identifying the junk food (knowledge of nutrition, nutrient content and balanced diet) and junk food and health problems. Among 30 respondents the Pre-tryout and try-out were conducted. Test items were analyzed by difficulty index and discrimination index. The FleschKincaid readability test was employed to measure the difficulty level of a sentence in English to understand it. Views from experts from various fields were taken to determine the content validity, construct validity and face validity of the questionnaire. The final questionnaire 
retained 31 items depending on the rejection criterion. The reliability of questionnaire was found high and it was established by test-retest method.

The knowledge questionnaire regarding junk food consumption has a great potentiality in the prevention of health hazards due to consumption of junk foods. These questionnaires will help to find out the level of knowledge of people of different categories regarding junk food and its effects. This knowledge will help to set the primary preventive measures for the health hazards due to junk food consumption. It may be used to aware, sensitize people regarding junk food. People themselves also will explore the information regarding junk foods and their health hazards while answering the questions. This will help them to be aware and encouraged to take care of themselves and young ones.

\section{Acknowledgement: None}

\section{Conflict of Interest: None}

\section{Source of Funding: None}

\section{Ethical Approval: Approved}

\section{REFERENCES}

1. Kashyap R, Joglekar A, and Verma, S. Effect of junk food on physical performance of school children. Indian Journal of Scientific Research and Technology. 2014; 2(4): 21-25.

2. Mandal A, Mandal GC. Prevalence of overweight and obesity among the urban adolescent English Medium School girls of Kolkata, India. Italian Journal of Public Health. 2012; 9(3).

3. Krishnaswamy K, Dietary Guidelines for Indians - A Manual. 2nd ed. Hyderabad, India: National Institute of Nutrition, ICMR, 2011.

4. Sharma V. Adolescents Knowledge Regarding Harmful Effects of Junk Food. IOSR Journal of Nursing and Health Science (IOSR-JNHS). 2013; 1(6): 01-04.

5. Buzescu A, Cristea AN, Avram L, Chiriţă C.The addictive behaviour induced by food monosodium glutamate. Experimental study. Romanian Journal Of Medical Practice. 2013; 8(4): 229-233.

6. Pearson N, Biddle SJ. Sedentary behavior and dietary intake in children, adolescents, and adults: a systematic review. American Journal of Preventive Medicine. 2011; 41(2):178-88.

7. Johnson S, Sahu R, Saxena P, Mathur HB, Agarwal HC. Nutritional Analysis of junk food. Report Published by Centre for Science and Environment. 2012; 1-23.

8. Shrivastav M, S Thomas. "Snack Consumption among Underprivileged Adolescent Girls.” INDIAN PEDIATRICS. 2012; 47: 888-90.

9. Puri S, Bhatia V, Swami H, Rai S, Mangat C. Impact of a diet and Nutrition Related Education Package On The Awareness And Practices Of School Children Of Chandigarh. International Scientific Journal. 2007; 6(1).

10. Majumdar B. Junk food, no play make city kids fat. 2005 May 27; Retrieved January 15, 2019, from https://www.telegraphindia.com/states/westbengal/junk-food-no-play-make-city-kidsfat/cid/1274126.

11. Press Trust of India. Ban sale of junk food on campuses, UGC directs varsities, colleges. The Times of India. 2018 August 22.

12. Calcutta University, Presidency takes steps to ban junk food sale on campus. India Today. 2018 August 27; Retrieved from https://www.indiatoday.in , Education Today > News.

13. Siniscalco TM, Auriat N. Quantitative research methods in educational planning. Paris: UNESCO International Institute for Educational Planning, 2005.

14. Creswell JW, Educational Research Planning, Conducting, and Evaluating Quantitative and Qualitative Research. 4th ed. Pearson Education Limited: Boston, United States of America, 2012; 399-40.

15. Kaliyaperumal K. Guideline for conducting a knowledge, attitude and practice (KAP) study. Community Ophthalmology. 2004; 4(1): 7-9.

16. Flesch R. How to Write Plain English: A Book for Lawyers and Consumers. Harper and Row. 1979; Retrieved from http://pages.stern.nyu.edu / / wstarbuc/ Writing/ Flesch.htm. 
17. Salkind N. Encyclopedia of Research Design. SAGE Publications: California, United States. 2010; Inc. https://doi.org/10.4135/9781412961288.

18. Ebel RL.The relation of item discrimination to test reliability. Journal of Educational Measurement. 1967; 4(3): 125-128.

19. Pongkiatchai R, Chongsuwat R, Howteerakul N, Pavadhgul P, Ollier W, Lophatananon A. Evaluation of a questionnaire to assess nutritional knowledge, attitudes and practices in a Thai population. Nutrition Journal. 2019; 18(35).

20. Parmenter K, Wardle J. Development of a general nutrition knowledge for adults.
European Journal of Clinical Nutrition. 1999; 53: 298-308.

21. Trakman GL, Eorsyth A, Hoye R, Belski R. Developing and validating a nutrition Knowledge questionnaire: key methods and considerations. Public Health Nutrition. 2017; 20(15): 2670-2679.

How to cite this article: Mondal SS, Mukherjee S, Sikdar DP. Construction of knowledge questionnaire regarding junk food consumption. Int J Health Sci Res. 2021; 11(6): 107-114. DOI: https://doi.org/10.52403/ijhsr.20210615 